\title{
Re: Cerebroplacental ratio in predicting adverse perinatal outcome: a meta-analysis of individual participant data. (First comment on BJOG-19-1584.R2)
}

\author{
Jose Morales-Rosello ${ }^{1}$, Gabriela Loscalzo ${ }^{2}$, Silvia Buongiorno ${ }^{2}$, Vaidilè Jakaitè ${ }^{2}$, and \\ Alfredo Perales-Marin ${ }^{1}$ \\ ${ }^{1}$ Hospital Universitario y Politecnico La Fe \\ ${ }^{2}$ Hospital Universitario y Politécnico La Fe
}

May 14, 2020

Letter to the Editor, BJOG Exchange

Re: Cerebroplacental ratio in predicting adverse perinatal outcome: a meta-analysis of individual participant data

\section{Dear Sir:}

We read with interest the study by Vollgraff Heidweiller-Schreurs et $\mathrm{al}^{1}$ in which the authors compare the abilities of the umbilical Doppler, middle cerebral Doppler and cerebroplacental ratio (CPR) for the prediction of adverse perinatal outcome (APO). We would like to congratulate the authors for this collaborative study and also for the CPR IPD initiative. However, we disagree with the conclusions and methodology upon which we would like to make several comments:

First, and foremost the main diagnostic performance of CPR relies on its ability to detect compromised fetuses unable to stand the stress of labor ${ }^{2}$. However, in absence of contractions, even endangered fetuses, with abnormal Doppler, may be delivered with a good APO and absence of acidosis. Second, CPR performance is strikingly dependent on the interval to labor, decreasing importantly after 15 days. If we consider that, beyond the appropriate performance range, Doppler techniques with different abilities may resemble equally accurate, the interval information becomes of crucial importance ${ }^{3}$. Third, as earlier published and regardless of CPR, the possibility of APO varies depending on the kind of labor onset. As a consequence, inductions with poor Bishop score are more likely to present acidosis and APO than deliveries with spontaneous onset and good obstetrics conditions ${ }^{4}$. All this implies that in order to perform appropriate comparisons between different Doppler techniques, cases with elective cesarean sections and higher intervals to labor should be discarded while the type of labor onset should be taken into account. However, as far as we are concerned none of this was done. Last but not least, when preterm fetuses are studied, admission to pediatric care units can be the result not only of hypoxia, but also of prematurity. Consequently, considering that in this scenario intrapartum and neonatal $\mathrm{pH}$ become the only accurate parameters to establish a clear diagnosis of acidosis, these should be homogeneous and obtained using similar $\mathrm{pH}$ thresholds among all cases studies. Unfortunately, this information was not provided.

Overlapping ROC curves may be the result of a similar performance between the studied parameters. However, considering the above-mentioned aspects they might also be the result of biased comparisons performed out of the appropriate performance ranges. While the findings of this meta-analysis do not support the use of CPR out of a research protocol, we would still like to consider that further research is needed to reach such conclusion, and that CPR remains as a poor predictor, but still the best individual predictor of APO. 
José Morales-Roselló*/**, Gabriela Loscalzo*, Silvia Buongiorno*, Vaidilè Jakaitė*, Alfredo PeralesMarín*/**

*Servicio de Obstetricia, Hospital Universitario y Politécnico La Fe, Valencia, Spain, and **Departamento de Pediatría, Obstetricia y Ginecología, Universidad de Valencia, Valencia, Spain.

\section{REFERENCES}

1. Vollgraff Heidweiller-Schreurs CA, van Osch IR, Heymans MW, Ganzevoort W, Schoonmade LJ, Bax CJ, Mol BWJ, de Groot CJ, Bossuyt PM, de Boer MA; CPR IPD Study Group. Cerebroplacental ratio in predicting adverse perinatal outcome: a meta-analysis of individual participant data. BJOG. 2020 May 3. doi: 10.1111/1471-0528.16287.

2. Khalil AA, Morales-Rosello J, Morlando M, Hannan H, Bhide A, Papageorghiou A, Thilaganathan B. Is fetal cerebroplacental ratio an independent predictor of intrapartum fetal compromise and neonatal unit admission? Am J Obstet Gynecol. 2015 Jul;213(1):54.e1-54.e10.

3. Akolekar R, Syngelaki A, Gallo DM, Poon LC, Nicolaides KH. Umbilical and fetal middle cerebral artery Doppler at 35-37 weeks' gestation in the prediction of adverse perinatal outcome. Ultrasound Obstet Gynecol. 2015 Jul;46(1):82-92.

4. Garcia-Simon R, Figueras F, Savchev S, Fabre E, Gratacos E, Oros D. Cervical condition and fetal cerebral Doppler as determinants of adverse perinatal outcome after labor induction for late-onset small-forgestational-age fetuses. Ultrasound Obstet Gynecol. 2015 Dec;46(6):713-7. 